\title{
BMJ Open Dimensions of tinnitus-related complaints reported by patients and their significant others: protocol for a systematic review
}

\author{
Haúla Haider, ${ }^{1}$ Kathryn Fackrell, ${ }^{2,3}$ Veronica Kennedy, ${ }^{4}$ Deborah A Hall ${ }^{2,3}$
}

To cite: Haider H, Fackrell K, Kennedy V, et al. Dimensions of tinnitus-related complaints reported by patients and their significant others: protocol for a systematic review. BMJ Open 2016;6:e009171. doi:10.1136/bmjopen-2015009171

- Prepublication history for this paper is available online. To view these files please visit the journal online (http://dx.doi.org/10.1136/ bmjopen-2015-009171).

Received 22 June 2015 Revised 11 January 2016 Accepted 11 April 2016

\section{CrossMark}

${ }^{1}$ ENT Department, Hospital Cuf Infante Santo-Nova Medical School, Lisbon, Portugal

${ }^{2}$ Nottingham Hearing Biomedical Research Unit, National Institute for Health Research (NIHR), Nottingham, UK

${ }^{3}$ Otology and Hearing group, Division of Clinical Neuroscience, School of Medicine, University of Nottingham, Nottingham, UK ${ }^{4}$ Department of Audiovestibular Medicine, Halliwell Health and Children's Centre, Bolton NHS Foundation Trust, Bolton, UK

\section{Correspondence to} Professor Deborah A Hall; deborah.hall@nottingham. ac.uk

\section{ABSTRACT}

Introduction: Over 70 million people in Europe and $>50$ million people in the USA are reported to experience tinnitus (the sensation of noise in the absence of any corresponding sound source). Tinnitus is a multidimensional concept. Individual patients may report different profiles of tinnitus-related symptoms which may each require a tailored management approach and an appropriate measure of therapeutic benefit. This systematic review concerns the patient perspective and has the purpose to find what symptoms are reported by people who experience tinnitus and by their significant others.

Methods and analysis: This protocol lays out the methodology to define what dimensions of tinnitusrelated symptoms patients and their significant others report as being a problem. Methods are defined according to the Preferred Reporting Items for Systematic reviews and Meta-analyses for Protocols (PRISMA-P) 2015 and data will be collated in a narrative synthesis. Findings will contribute to the eventual establishment of a Core Domain Set for clinical trials of tinnitus.

Ethics and dissemination: No ethical issues are foreseen. Findings will be reported at national and international ENT and audiology conferences and in a peer-reviewed journal.

Trial registration number: CRD42015020629.

\section{INTRODUCTION}

Tinnitus is a symptom-a ringing, buzzing or hissing sound perceived in the ears or head. Most cases of tinnitus are subjective, meaning that tinnitus is perceived only by the patient and there is no clinically identifiable source of the sound, and so assessing tinnitus is reliant on self-report measures. Tinnitus has been associated with a diverse range of complaints, including perceived loudness, sleep problems, existence of additional somatic symptoms, effects on daily life and on general health. ${ }^{1-3}$ Tinnitus may also have negative effects on psychological well-

\section{Strengths and limitations of this study}

- The protocol addresses the important question about what people (and their significant others) consider to be the problems related to the tinnitus. These domains can be used to inform choice of outcomes to assess therapeutic benefit.

- The review will collate independent evidence from patient stakeholder groups and actively seeks to reflect an international perspective.

- The review has a clearly established purpose and well-defined methods for data collection and synthesis.

- Limitations include an anticipated bias towards the UK and USA where questionnaires assessing the functional impact of tinnitus have predominantly been developed and an exclusion of reported symptoms where they have not been sorted into domain groupings.

being and personal quality of life, as well as a societal impact in terms of social withdrawal, impaired work performance and suchlike. ${ }^{45}$ These examples illustrate how tinnitus is a multidimensional concept. Each of these complaints has the potential to be defined as a domain: a distinct element (or dimension) of tinnitus such as how loud or how emotionally distressing a patient may find his or her tinnitus. In line with other chronic health conditions, these domains can be construed within the WHO's biopsychosocial model of disability relating to impairments, activity limitations and participation restrictions, as well as the environmental factors which affect these experiences. ${ }^{6}$

Self-report measures are used in clinical practice and in research to identify specific areas of a patient's life that are affected by tinnitus (informing the diagnosis), as well as to monitor a patient's progress with a particular treatment (determining the evaluation). However, there is growing acknowledgement 
that the heterogeneous nature of tinnitus complaints makes clinical research and outcome measurement difficult. For example, a compilation of the multi-item tinnitus questionnaires that have been published over the past few decades indicates at least 29 different instruments. These all purport to measure tinnitus 'severity', but do so using different questions, rating scales and subscales. Mostly they have been developed for clinical intake assessment to facilitate 'doctor-patient' decision-making about treatment goals and options. Few have been optimised for the evaluation of treatment-related change. ${ }^{78}$

Box 1 reports a list, and presents these instruments in chronological order. It is probably fair to say that none of the existing questionnaires covers all of the domains of tinnitus-related complaints. Questionnaire developers draw on clinical experience, but provide limited information in their publications on precisely how they established that the included domains and items are important to patients. There is no current consensus. Some questionnaires are clearly targeted towards the

Box 1 Multi-item tinnitus questionnaires (first author, date of publication)

Tinnitus Questionnaire/Tinnitus Effects Questionnaire (Richard Hallam, 1988). ${ }^{9}$

Tinnitus Handicap Questionnaire (Francis Kuk, 1990). ${ }^{10}$

Tinnitus Severity Scale (Robert Sweetow, 1990). ${ }^{11}$

Subjective Tinnitus Severity Scale (Jonathan Halford, 1991). ${ }^{12}$

Tinnitus Reaction Questionnaire (Peter Wilson, 1991). ${ }^{13}$

Tinnitus Severity Grading (Ross Coles, 1992). ${ }^{14}$

Tinnitus Handicap/Support Scale (Soly Erlandsson, 1992). ${ }^{15}$

Tinnitus Severity Index (Mary Meikle, 1995). ${ }^{16}$

Tinnitus Coping Style Questionnaire (Richard Budd, 1996). ${ }^{17}$

Tinnitus Handicap Inventory (Craig Newman, 1996). ${ }^{18}$

Tinnitus Cognitions Questionnaire (Peter Wilson, 1998). ${ }^{19}$

Intake Interview for Tinnitus Retraining Therapy (Margaret Jastreboff, 1999). ${ }^{20}$

Tinnitus Disability Questionnaire (Karoline Greimel, 1999). ${ }^{21}$

Structured Tinnitus-Interview (Wolfgang Hiller, 1999). ${ }^{22}$

Psychological Impact of Tinnitus Interview (Jane Henry, 2001). ${ }^{23}$

International Tinnitus Inventory (Veronica Kennedy, 2005). ${ }^{24}$

Tinnitus Experience Questionnaire (Carol Bauer, 2006). ${ }^{25}$

Chronic Tinnitus Acceptance Questionnaire (Jessica Moreland, 2007). ${ }^{26}$

Tinnitus Acceptance Questionnaire (Vendela Westin, 2008). ${ }^{27}$

Fear of Tinnitus Questionnaire (Rilana Cima, 2011) ${ }^{28}$

Tinnitus Catastrophizing Scale (Rilana Cima, 2011). ${ }^{28}$

Tinnitus Vigilance and Awareness Questionnaire (Rilana Cima, 2011). ${ }^{28}$

Self-Efficacy for Tinnitus Management Questionnaire (Sheri Smith, 2011). ${ }^{29}$

Tinnitus Fear Avoidance Scale (Maria Kleinstäuber, 2012). ${ }^{30}$

Tinnitus Functional Index (Mary Meikle, 2012). ${ }^{31}$

Attention and Performance Self-Assessment Scale (APSA) (Ulli Bankstahl, 2013). ${ }^{32}$

Tinnitus Magnitude Index (Caroline Schmidt, 2014). ${ }^{33}$

Tinnitus Primary Function Questionnaire (Richard Tyler, 2014). ${ }^{34}$

Tinnitus and Hearing Survey (James Henry, 2015). ${ }^{35}$ measurement of distinct domains (eg, Fear of Tinnitus Questionnaire; ${ }^{28}$ Self-Efficacy for Tinnitus Management Questionnaire ${ }^{29}$ ), whereas others are clearly targeted towards the measurement of the overall concept of tinnitus severity (eg, Tinnitus Questionnaire $;^{9}$ Tinnitus Functional Index $\left.{ }^{31}\right)$. Tyler et $a l^{34}$ have distinguished between primary and secondary effects of tinnitus, claiming that only four primary activities impaired by tinnitus (namely emotions, hearing, sleep and concentration) are relevant for measuring therapeutic changes, at least in such a way that those effects could be specifically attributed to a tinnitus-specific intervention. Patient views and/or opinions were not included in establishing these primary activities and therefore the importance of these domains warrants further investigation. Further research should be expanded to consider reporting of patient-reported complaints, whether they are universal or whether there are cultural differences. Other controversies have been debated. For example, with respect to quality of life, Newman $e t a l^{6}$ proposed that although psychometrically robust measures for tinnitus exist, there is no agreement about what tests should be included in the tinnitus assessment and how studies of health-related quality of life should be conducted.

A subgroup (COMiT, Core Outcome Measures in Tinnitus) working under the auspices of the COST Action European network for tinnitus (TINNET, http:// tinnet.tinnitusresearch.net/) is currently working collaboratively to establish an international standard for outcome measurements in clinical trials of tinnitus. ${ }^{36}$ Figure 1 illustrates the roadmap for the group. The first deliverable from that roadmap is expected to be a consensus on what outcome domains are essential (ie, core) to be captured in all controlled trials. These core domains will be identified as being important for the design of clinical trials assessing therapeutic benefit because they characterise those tinnitus-related complaints that are relevant to patients and their significant others. The procedures used to identify those core domains will reflect the perspectives of professionals and lay people, alike. This article therefore describes a systematic review protocol of the lay perspective (a first component of stage 1 of that roadmap) that relates to the domains of tinnitus-related symptoms reported by patients and their significant others. Another component of stage 1 includes a companion systematic review of what outcome domains have been evaluated in contemporary clinical trials of interventions for tinnitus. ${ }^{37}$

The opinions of people who experience tinnitus are particularly important because it is this group who tell us what aspects of tinnitus they find problematic and who will experience the benefits and adverse effects of treatments. For example, a recent collaboration with clinical professionals involved people with tinnitus in identifying and prioritising treatment uncertainties and in defining research questions relating to the treatment of tinnitus. ${ }^{38}$ There are many levels in which tinnitus can affect daily lives not only for the person 
Stage 1: Identify and agree on outcome domains

\begin{tabular}{|l|l|}
\hline $\begin{array}{l}\text { Systematic review of } \\
\text { controlled trials on } \\
\text { treatment effectiveness } \\
\text { (outcome domains) }\end{array}$ & $\begin{array}{l}\text { Review and narrative } \\
\text { synthesis based on } \\
\text { patient complaints }\end{array}$ \\
\hline
\end{tabular}

Delphi survey Round 1

Scoring the importance of domains for treatment effectiveness

Adding other important domains for further scoring of relevance Exclude nonimportant domains

\section{Delphi survey Round 2}

Voting on any proposed aggregation of overlapping domains Scoring the importance of domains for treatment effectiveness 作

Delphi survey Round 3
Feedback median scores for each stakeholder group to
participants
Scoring the relevance of domains for treatment effectiveness
Decision on domains to be included in COS

Output: Core domain set

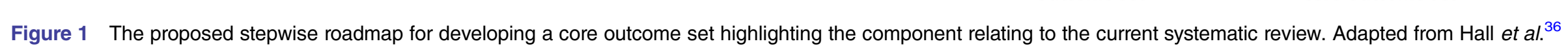

Stage 2: Identify and agree on outcome instrument

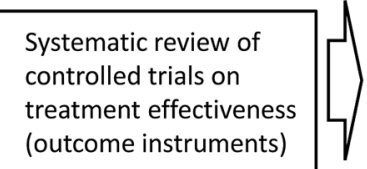

Evaluate instruments according to COSMIN checklist?

(outcome instruments)

\section{Systematic appraisal of instruments}

content validity

Structural validity

Internal consistency

Test-retest reliability

Sensitivity to treatment-

related change

Include in cos

- Include in preliminary cos

- Well-founded exclusion,

feasibly protected) (epyright

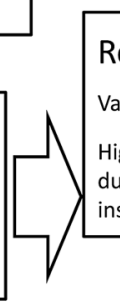

Output: Preliminary core outcome set

\section{Recommendation for validation}

Validation of the instrument

instruments
Stage 3: Prospective

validation of potentia

outcome instruments

\begin{tabular}{|l|l|}
\hline $\begin{array}{l}\text { Further research due to } \\
\text { lack of or low quality } \\
\text { validation studies on } \\
\text { domain instruments }\end{array}$
\end{tabular}

Stage 4: Finalise Core Stage 4: Finalise Cor
Outcome Set (COS)

\section{Output: Agreed Core \\ Outcome Set \\ Output: Contemporary \\ Core Outcome Set}

Vhe light any gaps where there are further research questions

to lack of or low quality validation studies on domain

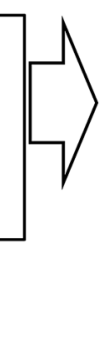


experiencing tinnitus but for those around them as well. Many tinnitus questionnaires ask about the impact of tinnitus on relationships, social activities and work. For example, the Tinnitus Handicap Inventory asks 'Do you feel that your tinnitus has placed stress on your relationships with members of your family and/or friends?'. ${ }^{18}$ The views and experiences of significant others, close relatives, friends, colleagues and family therefore would provide further insight into the reactions to tinnitus. People should also have opportunities to contribute to any consensus decisions about what are the most important outcome domains to be measured in such research studies. Ideally, one would seek a representative sample for interview to include a diversity of cultural and socioeconomic groups through purposive sampling. ${ }^{39}$ However, acknowledging our limited resources, the COMiT initiative agreed to take a pragmatic approach and to conduct a search to find where others might have already completed such work.

The longer term aim for the findings from this systematic review is therefore to contribute to the development of a core domain set for future controlled trials on tinnitus treatment effectiveness using data collected from people who experience tinnitus and their significant others. Text-based data in particular represents the lay perspective. The objective here is to establish which domains of functioning and disability arising from tinnitus are reported by people who experience the condition because they exert an important impact on their everyday personal life. This objective translates into the primary research question: What are the domains reported by people who experience tinnitus and their significant others which relate to why tinnitus is a problem for them?'.

Secondary research questions include:

- whether patients and significant others have similar or different perspectives;

- whether common clinical phenotypes influence the nature of the reported tinnitus complaints;

- whether health-related comorbidities influences the nature of the reported tinnitus complaints.

\section{METHODS AND ANALYSIS}

Methods are reported according to the Preferred Reporting Items for Systematic reviews and Meta-analyses for Protocols 2015 (PRISMA-P ${ }^{40}{ }^{41}$ ). Subheadings correspond to some of the items in the PRISMA-P checklist. The allocation of specific roles to named authors of the review will be made at a later date, and this information will clearly be acknowledged in any subsequent dissemination of findings.

\section{Eligibility criteria}

Study designs

Relevant work includes questionnaires, interviews, focus groups and web-based patient discussion forums. Information will be collected from any study type (including surveys, clinical trials and case series). Exclusion criteria are as follows: (1) studies of those medical conditions in which tinnitus is not the primary complaint, such as Ménière's disease, otosclerosis, chronic otitis media and generalised psychiatric comorbidities; (2) any studies whose main focus is on predicting tinnitus severity using regression modelling, rather than measuring tinnitus severity; (3) where individual complaints are reported, rather than having been collected and sorted into domains; (4) review articles and (5) any sources reporting expert opinions, manufacturers' articles, practice guidelines and case reports due to their more limited clinical and scientific value.

\section{Participants}

We will include men and women who report tinnitus as a primary symptom, as well as including their significant others. The age of the study sample will be $\geq 18$ years. Participants may represent clinical or non-clinical samples.

\section{Intervention}

The review does not specifically target intervention studies, but may refer to data that are collected as part of the initial assessment. Equally we will consider crosssectional, non-intervention studies.

\section{Comparison}

This review does not aim to evaluate specific interventions and as such no comparisons are appropriate or relevant.

\section{Outcomes}

The review does not specify treatment-related outcomes, but we will consider any types of tinnitus-related problems that are highlighted by patients and their significant others as being potential targets for therapeutic intervention.

\section{Timing}

All included work will be conducted after January 1980 . The rationale is that during the 1980 s, many interviews and preliminary questionnaires were administered to relatively large numbers of tinnitus preceding development of a number of the multi-item questionnaires that are now being used to evaluate functional, emotional and other effects of tinnitus. ${ }^{3}$

\section{Settings}

Articles will be included from research being conducted in any type of setting, such as academic sites where participants are recruited from the general public, nonclinical and occupational groups, and clinical sites including primary and secondary care services.

\section{Language}

Articles will not be restricted to English language so that we avoid excluding grey literature that is written for a 
particular lay audience in their native (non-English) language. To facilitate translation, authors will invite members of the EU COST Action TINNET to contribute to the data collection and to summarise in English the relevant information from non-English language documents. At present, the TINNET network includes 29 participating EU countries. For articles written in non-European languages, we will seek to obtain translations by native language speakers recruited from a university setting.

\section{Information sources}

All written documents will be included through the most relevant electronic research databases: PubMed (National Center for Biotechnology Information), Embase (OVID) and CINAHL (EBSCO). Eligible grey literature will include conference papers, undergraduate and postgraduate dissertations, reports from professional organisations and website content. Grey literature databases that will be searched for additional written documents are Open Grey and PsycEXTRA. Dissertations will be searched using DART (for Europe), ProQuest Dissertations and Theses (USA), and Networked Digital Library of Theses and Dissertations (for other databases of named international countries). Conference proceedings will be searched using Cos Conference Papers Index (ProQuest) and Web of Science (Thomson Reuters). Google will be searched using the keywords page by page up to the point at which a page contains no eligible records. In order to seek any further eligible documents for inclusion, we will conduct a manual search of any review articles found through the electronic database search. Tinnitus associations representing patients will also be contacted by email to enquire about commissioned reports and other relevant official documents that may include tinnitus-related complaints. Those tinnitus associations identified by searching Google are listed in box 2.

To ensure literature saturation, we will circulate a bibliography of the included records to tinnitus measurement experts. These are defined as the first author of a published article (known to the authors) that reports the development of a novel multi-item tinnitus questionnaire. First authors are listed in box 1. The manual search and personal contact with tinnitus associations and identified tinnitus experts will be ongoing up to the end of the data collection phase.

\section{Search strategy}

The electronic database search strategy will require 'tinnitus' in the title, in conjunction with additional relevant search terms defined as relevant medical subject headings (MeSH) or text words wherever possible. The search terms for PubMed, Embase and CINAHL will be guided by: '(tinnitus) AND (problem OR complain* OR symptom)' OR '(tinnitus) AND (patient OR significant other OR partner OR family)' (table 1). For example, the search strategy for PubMed will be: ${ }^{(}((()((($problem

\section{Box 2 List of patient associations}

American Tinnitus Association.

Australian Tinnitus Association (New South Wales).

Austrian Tinnitus Association. *

Belgian Tinnitus Association.*

British Tinnitus Association.

Canadian Tinnitus Foundation.

Danish Tinnitus Association. *

Finnish Tinnitus Association. *

French Tinnitus Association.*

German Tinnitus Association.*

Gibraltar Hearing Impaired and Tinnitus Association.

Hungarian Tinnitus Association. *

Irish Tinnitus Association. *

Italian Tinnitus Association. *

Lithuanian Tinnitus Association. *

Netherlands Tinnitus Association. *

New Zealand Tinnitus and Hyperacusis Support Network.

Norwegian Tinnitus Association. *

Polish Tinnitus Association. *

Spanish Tinnitus Association.*

Swedish Tinnitus Association. *

Swiss Tinnitus Association.*

Tinnitus (South Australia)

Tinnitus Association of Victoria.

Turkish Tinnitus Association. *

${ }^{\star}$ Member of the European Federation of Tinnitus Associations.

[Title/Abstract]) OR complain*[Title/Abstract]) OR symptom[Title/Abstract]) AND ("1980"[Date-Publication]: "3000"[Date-Publication])) AND Humans[Mesh] AND adult $[\mathrm{MeSH}]))$ AND tinnitus[Title]) AND Humans [Mesh] AND adult $[\mathrm{MeSH}]))$ OR $\quad\left(\left(\left(()_{(() \text {patient }}\right.\right.\right.$ [Title/Abstract]) OR significant other[Title/Abstract]) OR partner[Title/Abstract]) OR family[Title/Abstract]) AND ("1980"[Date-Publication]: “3000"[DatePublication])) AND Humans[Mesh] AND adult[MeSH])) AND tinnitus[Title]) AND Humans[Mesh] AND adult $[\mathrm{MeSH}])$. This will be adapted to the syntax and subject headings of the other databases. The authors do not have access to a health information specialist with database searching skills, and so the most experienced researchers will conduct the search.

\section{Study records}

Data management

$\mathrm{HH}$ and $\mathrm{KF}$ will be responsible for data management and will have editorial rights. Identified records will be saved into a master file (using Endnote) that will enable records to be tracked through the screening and data collection process. A simple system of record annotation will be implemented to capture reasons for exclusion. Included records will be allocated a study ID code to link each record in the master file with its corresponding full text and data collection sheet. 
Table 1 Matrix of the search terms for PubMed, Embase and CINAHL

\begin{tabular}{ll}
$\begin{array}{l}\text { First category } \\
\text { (title) }\end{array}$ & Second category \\
\hline Tinnitus & $\begin{array}{l}\text { problem (inc. problem } \\
\text { identification) }\end{array}$ \\
& $\begin{array}{l}\text { complain*(inc. psychological } \\
\text { aspect/consumer) } \\
\text { symptom (inc. symptom } \\
\text { assessment/symptom) }\end{array}$
\end{tabular}

Filter (human/adult) date

\section{Third category}

patient (inc. patient care/patient assessment/ patient care planning/patient participation/ patient coding/patient information/patient decision-making/ patient preference/ patient satisfaction/patient worry) significant other (inc. family/spouse)

partner (inc. interpersonal communication)

family (inc. family/family attitude/family functioning/family relation/family assessment)

\section{Selection process}

Endnote will be used to facilitate screening and remove duplicate records that are being managed within this software system. The first selection step will consider the title information to determine inclusion according to the PICOS and other specified eligibility criteria. All included records will then be reviewed manually to remove any duplicate records, using author names and study title. The second selection step will consider the abstract (or full text for some grey literature sources such as websites) for all potentially relevant records appearing to meet the inclusion criteria or for which there is insufficient information in the title to make a clear decision. Following this, the third selection step will obtain and consider the full text which met inclusion criteria or where there is still any uncertainty in content. The order of initial steps (ie, title screen followed by duplicate removal) is preferred because the search terms are broadly defined and so we predict a large number of ineligible records and also because not all information sources are readily transferred into endnote, for automated duplicate removal. We will adopt the principle that two team members will always perform each key step independently for every record (ie, title screening, full-text screening and data collection). DAH will conduct the key steps for every record, while other individuals may differ. If any discrepancies cannot be resolved, then a third person, an author $(\mathrm{HH})$ not involved with the data screening, will make a judgement on the data entered and act as an arbitrator. Descriptive statistics on the levels of agreement between team members will be reported.

\section{Data collection process}

Data collection will be guided by an electronic form (excel spreadsheet) that will also be used to collate all the information. To ensure consistency across reviewers, a full set of guidance notes will be produced for the data collection procedure and calibration exercises will be conducted with new members of the review team prior to any individual contribution to this review. The sheet and the guidance notes will be developed and revised through at least two iterations of piloting across several review authors. Data collection will be conducted independently and in duplicate (two people) for every included record. Given the 35-year period of the search, we will not contact the corresponding author by email to seek clarification for any missing data.

\section{Data items}

The data collection sheet will include a list of fields relating to study population, trial design and relevant study findings. Full details are given in box 3. If any information is not reported, then 'not stated' will be recorded in the corresponding field.

\section{Outcomes and prioritisation}

The priority for data synthesis and reporting of findings will be the primary outcome which relates to the domains of tinnitus-related complaints reported by patients and their significant others.

\section{Risk of bias in individual studies}

Given that this systematic review is not concerned with the effects of an intervention for tinnitus, we will not conduct a risk of bias assessment.

\section{Data synthesis}

The main purpose of this systematic review is to identify the domains of tinnitus-related complaints reported by patients and their significant others. A narrative synthesis will be reported using all included records, with information presented in a table to summarise and explain the characteristics and findings. We will seek to preserve the original descriptive labels for domains wherever possible. However, we anticipate that authors of different studies may use different terminology to describe the same underlying theoretical construct. In these cases, we will look carefully at the examples or explanations given by the study authors for each domain of tinnitus-related complaints (see Data items, box 3), and then use this information to cluster together related concepts across studies and report them in grouping table. ${ }^{42}$ For example, Tyler et $a l^{34}$ refer to 'concentration', while 
Box 3 Data items for systematic review of the domains of tinnitus-related complaints reported by patients and their significant others

Descriptive checklist:

- Study ID code.

- Record title.

- Name and contact details of corresponding author.

- Country where study is conducted.

- Date of publication (year).

- Aim of study.

Study population:

- Patients.

- Significant others.

- Other (give details).

- Sample size.

Age characteristics (mean and SD).

Tinnitus-related description of study population:

- Duration.

- Intermittent or constant.

- Pulsatile or non-pulsatile.

- Severity.

- Any other subtypes.

- Any other health-related comorbidities in the study population.

- Study type:

- Survey.

- Intervention trial.

- Case series.

- Other (give details).

- Primary method for collecting individual tinnitus-related complaints:

- Questionnaire.

- Interview.

- Focus group.

- Other.

- What specific instructions were given or questions asked?

- Did the authors use open or closed questioning?

- Open.

- Closed.

- Open and closed questions (give details).

- Description of any other relevant data collection methods.

- Primary method for data synthesis.

- Dimensions describing tinnitus-related complaints.

- For each dimension, one example or explanation of what is the underlying theoretical construct.

- Notes (this optional field will be used to record any further comments that may be deemed informative).

Meikle $e t a l^{31}$ refer to 'cognitive interference' which includes problems with concentration. For transparency of reporting, all the information on the domain groupings and the associated questionnaire items will clearly be presented in a table so that others can scrutinise our 'grouping' decisions. Additionally for narrative textbased data, we will tabulate the evidence for the domain groupings, by providing examples of the narrative text associated with each domain. In this way, each domain that we propose can be traced back to the supporting evidence from all the included studies. A committee including all four coauthors and people with tinnitus $(n=4)$ will examine the choice of words used to describe the labelling of the domains. If there is $<70 \%$ consensus from the committee that the label is not appropriate for each grouping, then the grouping and labelling will be re-examined in an iterative process.

The narrative synthesis will explore the relationship and findings within and between the included studies as three secondary questions (if there are sufficient data). Main findings will be illustrated by tables.

- To address the secondary research question about whether patients and significant others have similar or different perspectives, we will compile the reported domains of tinnitus-related complaints split according to the study population.

- To address the secondary research question about whether tinnitus 'subtypes' influence the nature of the tinnitus complaints that are reported, we will present a table that compiles the reported domains of tinnitusrelated complaints split according to common clinical phenotypes of tinnitus (acute/chronic, intermittent/ constant, pulsatile/non-pulsatile and severity grading).

- To address the secondary research question about whether a health-related comorbidity influences the nature of the tinnitus complaints that are reported, we will present a table that compiles the reported domains of tinnitus-related complaints split according to the most common comorbidities relating to tinnitus (including mental health problems such as anxiety and depression, hyperacusis and hearing loss).

An exploratory data synthesis will generate a complete list of specific instructions that were given or questions that were asked by the authors to elicit the information from patients and their significant others.

\section{Confidence in cumulative evidence}

Three assessments of the quality of collecting, defining and reporting the domains of tinnitus-related complaints are planned (table 2):

- The first will ascertain whether the participants recruited reflect the heterogeneity of the typical clinical population with tinnitus. A yes/no decision will be based on a weighted composite assessment of the sample size, age characteristics (mean and SD) and any recruitment limitations by subgroup (eg, a specific comorbidity).

- The second evaluate the extent to which the authors used an open questioning format. This is important because closed questioning introduces authors' bias by imposing preconceptions on what sort of tinnitusrelated complaints people might consider important.

- We anticipate that a large number of studies will report text-based data, from which themes have been identified. Data analysis of narrative text requires specific skills within the study team and should be reported sufficiently clearly to enable replication. The third quality assessment will therefore assess whether studies report the competencies of those authors conducting the identification of themes. It 
Table 2 Quality items for the systematic review of the domains of tinnitus-related complaints reported by patients and their significant others

\begin{tabular}{llll}
\hline Quality checklist & \multicolumn{3}{l}{ Count of records } \\
\hline $\begin{array}{l}\text { Participant sample reflects } \\
\text { the heterogeneity of the }\end{array}$ & $\begin{array}{l}\text { Yes } \\
(n=)\end{array}$ & No $(n=)$ & \\
$\begin{array}{l}\text { tinnitus population } \\
\begin{array}{l}\text { Open questioning format } \\
\text { Yes }\end{array}\end{array}$ & $\begin{array}{l}\text { No, } \\
\text { mixed } \\
(n=)\end{array}$ & $\begin{array}{l}\text { No, } \\
\text { closed } \\
(n=)\end{array}$ \\
$\begin{array}{l}\text { Analysis methods reported } \\
\text { sufficiently clearly to } \\
\text { enable replication }\end{array}$ & $\begin{array}{l}\text { Yes } \\
(n=)\end{array}$ & No $(n=)$ & \\
\hline
\end{tabular}

will also seek to determine the proportion of studies for which the analysis methods have been reported sufficiently clearly to enable replication.

\section{Ethics and dissemination}

No ethical issues are foreseen. The findings will be reported at national and international ENT and audiology conferences and in a peer-reviewed journal using the Preferred Reporting Items for Systematic Reviews and Meta-Analyses (PRISMA, http://www.prismastatement.org/). Publication reporting will include the checklist and the flow diagram to depict the flow of information through the different phases of the systematic reviews. All data collected according to the data items will be available on request to the extent that it is not included in the published systematic review article.

Contributors HH is the guarantor of the review. DAH created the search strategies. $\mathrm{HH}$ and $\mathrm{DAH}$ contributed equally to all other stages of the protocol development and manuscript production. KF made a substantial scientific contribution to the revised manuscript. VK provided consultative and advice. $\mathrm{HH}, \mathrm{KF}, \mathrm{VK}$ and DAH approved the final manuscript.

Funding This work is supported by COST Action (TINNET BM1306). The COMiT initiative is partially supported through an independent research programme funded under the Biomedicine and Molecular Biosciences European Cooperation in Science and Technology (COST) Action framework. Travel, subsistence and accommodation for DAH to work with $\mathrm{HH}$ was provided through one Short Term Scientific Mission.

Disclaimer The views expressed are those of the authors and not the funder. Competing interests None declared.

Provenance and peer review Not commissioned; externally peer reviewed.

Data sharing statement All data collected according to the data items will be available on request to the extent that it is not included in the published systematic review article.

Open Access This is an Open Access article distributed in accordance with the terms of the Creative Commons Attribution (CC BY 4.0) license, which permits others to distribute, remix, adapt and build upon this work, for commercial use, provided the original work is properly cited. See: http:// creativecommons.org/licenses/by/4.0/

\section{REFERENCES}

1. Tyler RS, Baker LJ. Difficulties experienced by tinnitus sufferers. $J$ Speech Hear Disord 1983;48:150-4.
2. Stouffer JL, Tyler RS. Characterization of tinnitus by tinnitus patients. J Speech Hear Disord 1990;55:439-53.

3. Hoekstra CE, Wesdorp FM, van Zanten GA. Socio-demographic, health, and tinnitus related variables affecting tinnitus severity. Ear Hear 2014;35:544-54.

4. Kamalski DM, Hoekstra CE, van Zanten BG, et al. Measuring disease-specific health-related quality of life to evaluate treatment outcomes in tinnitus patients: a systematic review. Otolaryngol Head Neck Surg 2010;143:181-5.

5. Maes IH, Cima RF, Vlaeyen JW, et al. Tinnitus: a cost study. Ear Hear 2013;34:508-14.

6. Newman CW, Sandridge SA, Jacobson GP. Assessing outcomes of tinnitus intervention. J Am Acad Audiol 2014:25:76-105.

7. Meikle MB, Stewart BJ, Griest SE, et al. Tinnitus outcomes assessment. Trends Amplif 2008;12:223-35.

8. Fackrell K, Hall DA, Barry J, et al. Tools for tinnitus measurement: development and validity of questionnaires to assess handicap and treatment effects. Chapter 2. In: Signorelli F, Turiman F, eds. Tinnitus: causes, treatment and short and long-term health effects. Nova Science Publishers Inc, 2014:13-60.

9. Hallam RS, Jakes SC, Hinchcliffe R. Cognitive variables in tinnitus annoyance. Br J Clin Psychol 1988;27:213-22.

10. Kuk FK, Tyler RS, Russell D, et al. The psychometric properties of a tinnitus handicap questionnaire. Ear Hear 1990;11:434-45.

11. Sweetow RH, Levy MC. Tinnitus severity scaling for diagnostic/ therapeutic usage. Hear Instrum 1990;41:20-46.

12. Halford JBS, Anderson SD. Tinnitus severity measured by a subjective scale, audiometry and clinical judgement. J Laryngol Otol 1991;105:89-95.

13. Wilson PH, Henry J, Bowen M, et al. Tinnitus Reaction Questionnaire: psychometric properties of a measure of distress associated with tinnitus. J Speech Hear Res 1991;34:197-201.

14. Coles RRA, Lutman ME, Axelsson A, et al. Tinnitus severity gradings: Cross-sectional studies. In: Aran JM, Dauman R, eds. Tinnitus 91: Proceedings of the Fourth International Tinnitus Seminar. New York: Kugler, 1992:453-5.

15. Erlandsson SI, Hallberg LRM, Axelsson A. Psychological and audiological correlates of perceived tinnitus severity. Audiology 1992;31:168-79.

16. Meikle MB, Griest SE, Stewart BJ, et al. Measuring the negative impact of tinnitus: a brief severity index. Abstr Assoc Res Otolaryngol 1995;167.

17. Budd RJ, Pugh R. Tinnitus coping style and its relationship to tinnitus severity and emotional distress. J Psychosom Res 1996:41:327-35.

18. Newman CW, Jacobson GP, Spitzer JB. Development of the Tinnitus Handicap Inventory. Arch Otolaryngol Head Neck Surg 1996;122:143-8.

19. Wilson PH, Henry JL. Tinnitus Cognitions Questionnaire: development and psychometric properties of a measure of dysfunctional cognitions associated with tinnitus. Int Tinnitus $J$ 1998;4:23-30.

20. Jastreboff MM, Jastreboff PJ. Questionnaires for assessment of the patients and treatment outcome. In: Hazell J, ed. Proceedings of the Sixth International Tinnitus Seminar. London: Tinnitus and Hyperacusis Centre, 1999:487-90.

21. Greimel KV, Leibetseder M. Can tinnitus be measured? Methods for assessment of tinnitus-specific disability and presentation of the Tinnitus Disability Questionnaire. HNO 1999;47:196-201.

22. Hiller W, Goebel G, Schindelmann U. Developing a structured interview to assess audiological, aetiological and psychological variables of tinnitus. Proceedings of the Sixth International Tinnitus Seminar. London: The Tinnitus and Hyperacusis Centre, 1999:277-82.

23. Henry JL, Kangas M, Wilson PH. Development of the psychological impact of tinnitus interview: a clinician-administered measure of tinnitus-related distress. Int Tinnitus J 2001;7:20-6.

24. Kennedy V, Chéry-Croze S, Stephens D, et al. Development of the International Tinnitus Inventory (ITI): a patient-directed problem questionnaire. Audiol Med 2005;3:228-37.

25. Bauer CA, Brozoski TJ. Effect of gabapentin on the sensation and impact of tinnitus. Laryngoscope 2006;116:675e681.

26. Moreland J. Illness representations, acceptance, coping and psychological distress in chronic tinnitus [PhD thesis]. UK: University of Leicester, 2007.

27. Westin V, Hayes SC, Andersson G. Is it the sound or your relationship to it? The role of acceptance in predicting tinnitus impact. Behav Res Ther 2008;46:1259-65.

28. Cima RF, Crombez G, Vlaeyen JW. Catastrophizing and fear of tinnitus predict quality of life in patients with chronic tinnitus. Ear Hear 2011;32:634-41. 
29. Smith SL, Fagelson M. Development of the self-efficacy for tinnitus management questionnaire. J Am Acad Audiol 2011;22:424-40.

30. Kleinstäuber M, Jasper K, Schweda I, et al. The role of fear-avoidance cognitions and behaviors in patients with chronic tinnitus. Cogn Behav Ther 2013;42:84-99.

31. Meikle MB, Henry JA, Griest SE, et al. The Tinnitus Functional Index: development of a new clinical measure for chronic, intrusive tinnitus. Ear Hear 2012;33:153-76.

32. Bankstahl US, Görtelmeyer R. Measuring subjective complaints of attention and performance failures-development and psychometric validation in tinnitus of the self-assessment scale APSA. Health Qual Life Outcomes 2013;11:86.

33. Schmidt CJ, Kerns RD, Griest S, et al. Toward development of a Tinnitus Magnitude Index. Ear Hear 2014;35:476-84.

34. Tyler R, Ji H, Perreau A, et al. Development and validation of the Tinnitus Primary Function Questionnaire. Am J Audiol 2014;23:260-72.

35. Henry JA, Griest S, Zaugg TL, et al. Tinnitus and hearing survey: a screening tool to differentiate bothersome tinnitus from hearing difficulties. Am J Audiol 2015;24:66-77.

36. Hall DA, Haider H, Kikidis D, et al. Towards a global consensus on outcome measures for clinical trials in tinnitus: report from the first international meeting of the COMiT initiative 14 November 2014, Amsterdam, The Netherlands. Trends Hear 2015;19:1-7.
37. Hall DA, Szczepek A, Kennedy V, et al. Current reported outcome domains in studies of adults with a focus on the treatment of tinnitus: protocol for a systematic review. BMJ Open 2015;5: e009091.

38. Hall DA, Mohamad N, Firkins L, et al. Identifying and prioritizing unmet research questions for people with tinnitus: the James Lind Alliance Tinnitus Priority Setting Partnership. Clin Investig 2013;3:21-8.

39. Granberg S, Pronk M, Swanepoel DW, et al. The ICF core sets for hearing loss project: functioning and disability from the patient perspective. Int J Audiol 2014;53:777-86.

40. Moher D, Shamseer L, Clarke M, et al., PRISMA-P Group. Preferred reporting items for systematic review and meta-analysis protocols (PRISMA-P) 2015 statement. Syst Rev 2015;4:1.

41. Shamseer L, Moher D, Clarke M, et al., PRISMA-P Group. Preferred reporting items for systematic review and meta-analysis protocols (PRISMA-P) 2015: elaboration and explanation. BMJ 2015;349: g7647.

42. Popay J, Roberts H, Sowden A, et al. Guidance on the conduct of narrative synthesis in systematic reviews. A product from the ESRC methods programme. 2006, Version 1. Unpublished report. Lancaster University: Institute for Health Research, 2006. http://citeseerx.ist.psu.edu/viewdoc/download?doi=10.1.1.178. 3100\&rep=rep1\&type=pdf (accessed 4 Oct 2016) 